\title{
Single Sensor Interventional All-Optical Ultrasound Imaging: Beam Characteristics and Bandwidth Performance
}

\author{
Robert M Stafford-Williams ${ }^{*, \ddagger}$, Manish K Tiwari ${ }^{\dagger, \ddagger}$, Adrien E Desjardins*, ${ }^{*}$, Erwin J Alles ${ }^{*, \ddagger}$ \\ * Department of Medical Physics \& Biomedical Engineering, University College London, London, UK \\ ${ }^{\dagger}$ Nanoengineered Systems Laboratory, UCL Mechanical Engineering, University College London, London, UK \\ $\ddagger$ Wellcome / EPSRC Centre for Interventional and Surgical Sciences, University College London, London, UK
}

\begin{abstract}
Optical ultrasound is an emerging imaging paradigm that utilises optical fibres to emit and receive ultrasound. Their small lateral dimensions enable highly miniaturised imaging probes, for a range of interventional applications. This work presents a numerical study to determine the beam width, divergence angle and pressure retention for a range of probe geometries. Additionally, pulse-echo imaging of point scatterer targets is presented, and the impact of bandwidth filtering investigated. The results from this study provide the basis for developing a wide range of single sensor ultrasound devices.
\end{abstract}

Index Terms-Optical Ultrasound, Miniaturized, Single Sensor, Directional Ultrasound Sensor, Simulation

\section{INTRODUCTION}

Conventional ultrasound imaging probes typically consist of centimetre scale arrays of individual transducers that achieve sub-mm resolution [1]. In interventional applications, where access to millimetre-scale internal lumens and flexibility around curvatures within the body are required, use of such traditional arrays as single-use devices can be prohibitively expensive. This study explores the development of alternative imaging probes comprising a single, highly directional element. Basing such a device on high directionality obviates the need for an image reconstruction step, and thus reduces the associated sensitivity to position uncertainty due to potential motion artefacts [1].

By using optical ultrasound (OpUS), highly directional ultrasound imaging beams can be achieved for ultrasound image formation [2]. OpUS transmission utilises an optical fibre with a tip coated in a material that absorbs light from a source such as a pulsed laser [3]. On absorption, this material undergoes thermal expansion via the photoacoustic effect, and in doing so emits a pressure wave i.e. an acoustic wave into the surrounding medium around the fibre tip. The backreflected ultrasound wave from a scatterer within the medium is detected optically. Typically, Fabry-Pérot resonators are applied as OpUS detectors, which provide an omni-directional response and a high detection bandwidth [4].

For interventional applications, single-element optical ultrasound probes consisting of one transmitter and one receiver are

This work was supported by the Wellcome Trust (203145Z/16/Z), the Engineering and Physical Sciences Research Council (EPSRC) (NS/A000050/1, EP/N021177/1, EP/S001506/1), and the Rosetrees Trust (PGS19-2/10006). ideal due to their small lateral dimensions, and their imaging capability has been explored using several different paradigms. For instance, such probes have been incorporated into a robotmounted rigid instrument for 3D imaging of a phantom [5], and integrated into a commercial endoscope for free hand imaging aided by electro-magnetic tracking [2]. Both studies demonstrated high-quality imaging of placental phantoms with an imaging depth of $c a .3 \mathrm{~cm}$. Furthermore, rotational crosssectional imaging of an ex vivo swine carotid artery (in a fashion similar to intravascular ultrasound [IVUS]) has also been achieved using a single transmit-receive pair of sideemitting OpUS fibres [6]. Finally, a single-element OpUS probe has been demonstrated in vivo to guide a transeptal puncture procedure in a pre-clinical swine model [7].

This study builds on these successes in single-element OpUS imaging, to develop a novel imaging paradigm, where highly directional, side-emitting OpUS probes are rapidly actuated to generate real-time, 2D ultrasound imaging. Using directional imaging without image reconstruction enables a range of interventional applications, such as endobronchial, gastrointestinal and cardiovascular imaging. Here, numerical optimisation of the transducer surface geometry was performed to inform the design and development of single sensor ultrasound probes, exploring beam width and divergence, pressure retention, and imaging quality across a range of different parameters and bandwidths.

\section{Methods}

\section{A. Simulated probe design}

This study explores beam characteristics while considering two different transmitter probe designs. One design utilises side-emitting probes based on previous experiments [6] (Fig. 1), which achieved high-quality rotational ultrasound images from within blood vessel segments. The second design is obliquely-emitting, and uses fibres that are angle-polished and directly coated with an OpUS generator material typically comprised of an optical absorber embedded in PDMS [3]. However, for this design certain geometries will result in total internal reflection (TIR), in which case light would not reach the OpUS generating coating. The critical angle resulting in TIR when exceeded was calculated as $\theta_{c}=\arcsin \left(n_{2} / n_{1}\right)=$ $78.87^{\circ}$, where $n_{2}=1.43$ and $n_{1}=1.45705$ are the refractive 


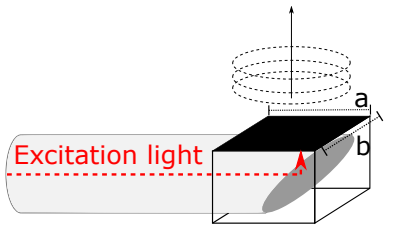

Direction of fibre actuation

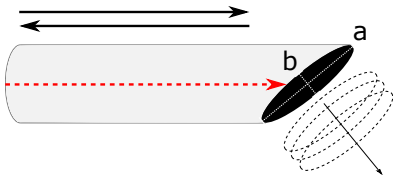

Fig. 1. Two probe designs for linear actuated OpUS. Left: excitation light is reflected to an OpUS generating coating deposited on the side of the fibre to achieve a side-emitting source (indicated by the dashed ellipses; adapted from [6]). Right: the OpUS coating is deposited onto an angle-polished end face directly to achieve an obliquely-emitting source.

indices of PDMS [8] and the core of a common multimode fibre [9] at a wavelength of $632.8 \mathrm{~nm}$. This threshold was used to label which transducer geometries are suited to which fabrication method.

\section{B. Simulation Parameters}

Forward pressure propagation was simulated using the FOCUS toolbox for MATLAB [10]. FOCUS uses computationally efficient closed-form solutions [11], [12] for the spatiotemporal response of rectangular piston transducers. While the probes effectively exhibit elliptical transmitter surfaces, these surfaces will be modelled as rectangles since no closed-form expression exists for an elliptical geometry. This approximation has previously shown good agreement with experimental OpUS results [13].

The temporal signature was modelled as a $15 \mathrm{MHz}$ tone burst of 3.5 cycles, sampled at a rate of $250 \mathrm{MHz}$ to match the experimental setup. The acoustic medium was modelled as lossless water, with a speed of sound of $1500 \mathrm{~m} / \mathrm{s}$. The pressure fields were sampled at $10 \mu \mathrm{m}$ per pixel over a $60 \mathrm{~mm}$ $\times 60 \mathrm{~mm}$ grid. The imaging aperture measured $12 \mathrm{~mm}$ and was sampled in $10 \mu \mathrm{m}$ steps. OpUS source geometries were considered with elevational and lateral dimensions ( $b$ and $a$, respectively) ranging between 100 and $1000 \mu \mathrm{m}$. Simulations were performed using MATLAB (2019a; The MathWorks, MA, USA) on a workstation with a Windows 10 Pro 64-bit operating system, with a $2.5 \mathrm{GHz}$ quad-core Intel ${ }^{\circledR}$ Core $^{\mathrm{TM}}$ i5-3210M CPU and 16GB RAM.

\section{Extraction of beam characteristics}

To study the performance of different source geometries, the following quantities were extracted. The beam width, and indicator of lateral resolution, was taken as the full-widthat-half-maximum (FWHM) of the maximum pressure in the plane $2 \mathrm{~mm}$ away parallel to the transducer surface. The divergence angle, which allows approximations of beam width at any given distance, was taken by calculating the angle between the apex of the FWHM measured at $2 \mathrm{~mm}$ and $30 \mathrm{~mm}$. The pressure retention at depth, which is broadly proportional to the imaging depth, was defined in this study as $100 \times\left(p_{30 \mathrm{~mm}} / p_{2 \mathrm{~mm}}\right)$, where $p_{30 \mathrm{~mm}}$ and $p_{2 \mathrm{~mm}}$ are the maximum pressure amplitude observed at a distance of $30 \mathrm{~mm}$ and $2 \mathrm{~mm}$ from the transducer surface, respectively.

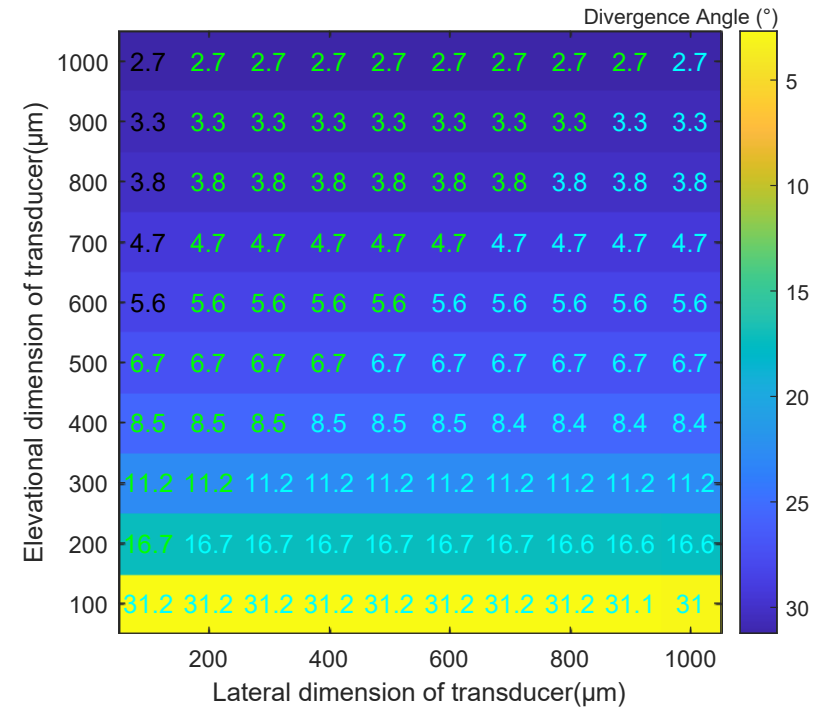

Fig. 2. Beam divergence (along the elevational axis) for a range lateral (a) and elevational (b) source dimensions. Elements printed in black are unattainable for an obliquely emitting source due to total internal reflection. Cyan elements cannot be achieved using angle polishing, hence only the elements indicated in green can be achieved using an obliquely emitting source. The side-emitting design can achieve all geometries studied. The divergence angle along the lateral axis is the transpose of this table.

\section{Directional imaging simulations}

To examine the imaging performance of single-probe transducers, pulse-echo images of a $3 \times 3$ grid of point scatterers were simulated. To create each image, the incident pressure wave at the location of the point scatterer was computed using the FOCUS toolbox. The scattered wave propagates back to the OpUS imaging probe, where it is detected optically. This detector is assumed to have an omnidirectional and spectrally uniform response [4], and as such can be modelled as a point detector through the three-dimension free-space Green's function [14],

$$
G_{3 D}=\frac{\delta\left(t-\frac{|\vec{x}|}{c}\right)}{4 \pi|\vec{x}|},
$$

where $\delta$ represents a Dirac delta distribution, $c$ is the speed of sound, and $\vec{x}$ is the vector between the source-receiver position and the scatterer. The pulse-echo time trace detected is given by the temporal convolution between the incident pressure and the Green's function at the location of the scatterer. This process is repeated, and all the time traces summed, for all point scatterers in the numerical phantom. This allows for an assessment of the axial and lateral imaging resolution of directional imaging. Images were obtained for small, medium and large transducer sizes, measuring $200 \mu \mathrm{m} \times 100 \mu \mathrm{m}$, $500 \mu \mathrm{m} \times 400 \mu \mathrm{m}$, and $1000 \mu \mathrm{m} \times 900 \mu \mathrm{m}$, respectively. Images were formed at a signal-to-noise ratio (SNR) of 15 , 20 and $25 \mathrm{~dB}$, and the effect of bandwidth on imaging was explored using high-pass elliptic filters with cut-on frequencies of $20 \mathrm{MHz}, 30 \mathrm{MHz}, 40 \mathrm{MHz}$ and $50 \mathrm{MHz}$. Finally, envelope detection and log-compression were applied. 


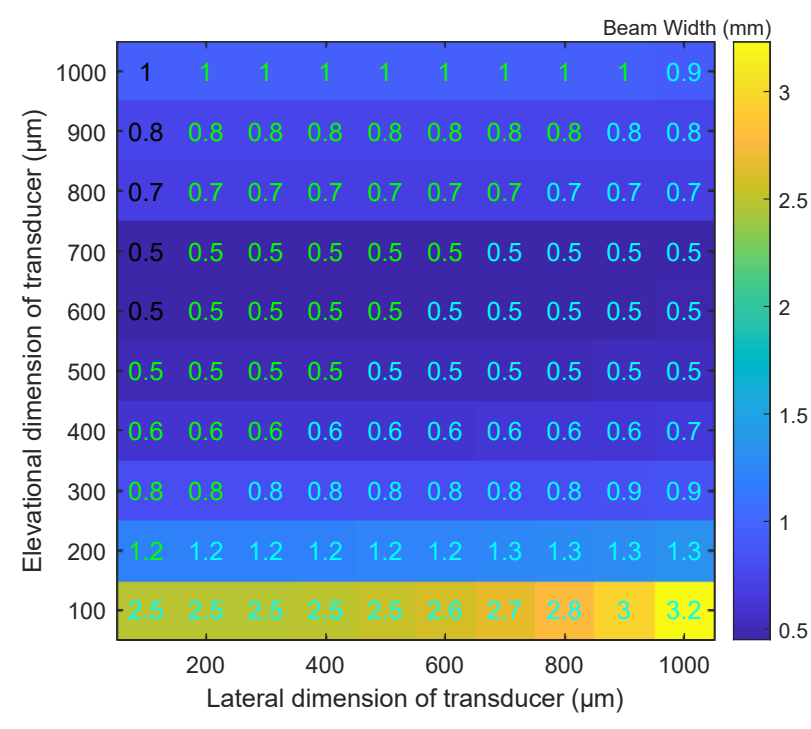

Fig. 3. Beam width (along elevational axis) for a range of source dimensions at an axial depth of $2 \mathbf{~ m m}$. Colour coding is identical to that in Fig. 2. The beam width along the lateral axis is the transpose of this table.

\section{RESULTS}

\section{A. Beam characteristics}

As a trend, the beam divergence angle decreases with increasing transducer dimension (Fig. 2). In contrast, the beam width increases with increasing transducer dimension (Fig. 3). This trend continues up to a source dimension of $700 \mu \mathrm{m}$, beyond which the beam width increases and approximately matches the transducer size. This limiting size is dictated by the spectral response of the source, and would be different for different temporal signatures. Pressure retention at depth is largest (up to 25\%) for larger transducer dimensions (Fig. 4). For the smallest transducer dimensions considered, this ratio is only $7 \%$.

\section{B. Directional imaging and bandwidth performance}

Due to a greater directivity and better pressure retention, larger transducers provide better contrast and can visualise point scatterers at greater depths (Fig. 5). In addition, the spatial extent of the point-spread function is smaller for larger transducers, resulting in a reduction of the "wingshaped" artefacts observed. Applying high-pass filters with increasing cut-on frequencies results in better lateral resolution and reduced artefacts, at the expense of reduced contrast and imaging depth.

\section{DISCUSSION AND CONCLUSION}

This study has provided fundamental insights regarding the impact of transducer dimensions on ultrasound beam characteristics. The parameter sweep performed allows for the optimisation of designs for future imaging probes. The minimum size of beam width being shown at $700 \mu \mathrm{m}$ gives us a target value for maximal beam directionality for single sensor probes. These values can also inform the design of conventional, nonOpUS ultrasound transducers, with applications for designing

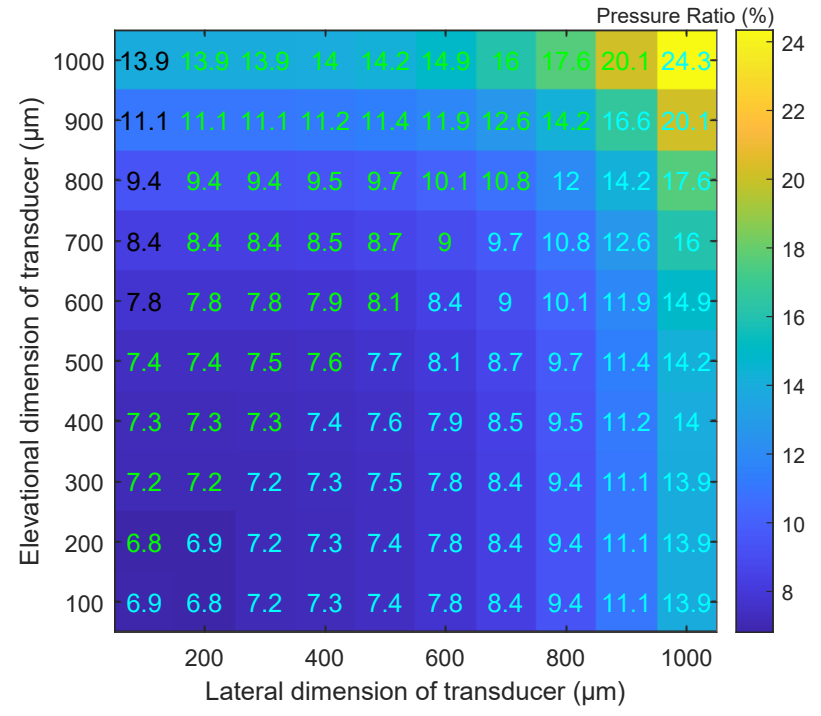

Fig. 4. Pressure retention at depth for a range of source dimensions. Colour coding is identical to that in Fig. 2.

transmitters of similar size but different materials such as piezoelectric materials, CMUTs and others.

The imaging simulations demonstrated the effect of transducer size on imaging quality, as well as the trade-off between resolution and contrast when filtering out an increasing proportion of the lower frequency components, for which source directivity is low. Conventionally, OpUS imaging is performed using pulsed light sources. In this case, frequency filtering could be performed in post-processing, at the expense of a reduction in SNR. This decrease in SNR can be mitigated using modulated light sources, which can confine the energy to just the desired frequency band [15].

Using the FOCUS toolbox imposes limits on the accuracy of the simulations due to the assumption of a homogeneous medium and a rectangular approximation of the transducer geometry. Whilst there has been strong agreement between FOCUS simulations and experimental OpUS data in previous studies, work further validating the particular set of data against experimental results will be explored in future work. In this study, the temporal signature of the source and the SNR of the system have been modelled on typical values observed from similar OpUS transducers. However, in future, these parameters could be matched to those measured from physical individual transducers.

Exploring the beam characteristics and bandwidth performance of miniature ultrasound sensors has yielded a useful set of data to guide the development of miniature OpUS probes capable of video-rate 2D imaging at a probe diameter of less than $1 \mathrm{~mm}$. Alongside future validation studies, further work on incorporating more complex image reconstruction techniques (such as delay-and-sum) into the simulation will provide a broad framework for exploring a wide variety of optical ultrasound device development design. 


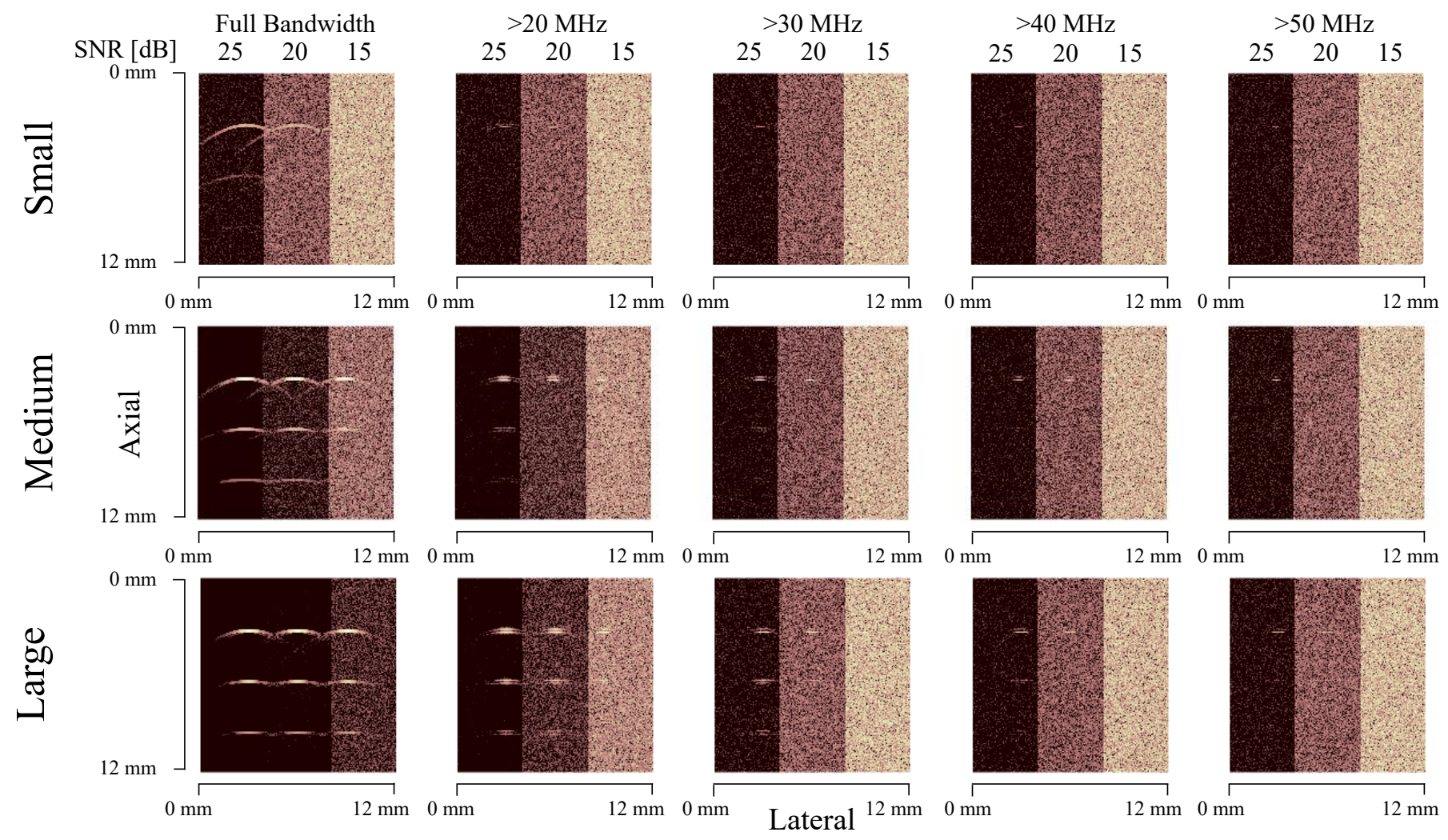

Fig. 5. Simulated images of directional ultrasound transducers. Top row: source geometry "small" (200 $\mu$ m elevational $\times 100 \mu \mathrm{m}$ lateral). Middle row: source geometry "medium" (500 $\mu \mathrm{m}$ elevational $\times 400 \mu \mathrm{m}$ lateral). Bottom row: source geometry "large" (1000 $\mu \mathrm{m}$ elevational $\times 900 \mu \mathrm{m}$ lateral). Each panel displays composite images obtained with three different SNRs, and in each column a different cut-on frequency of the high pass filter is applied to further improve source directivity.

\section{REFERENCES}

[1] T.L. Szabo. Diagnostic Imaging: Inside Out. Boston, Mass. ; London : Elsevier Academic Press, 2004.

[2] Erwin J Alles, George Dwyer, Richard J Colchester, Efthymios Maneas, Danail Stoyanov, and Adrien E Desjardins. Real-time and Freehand Multimodal Imaging: Combining White Light Endoscopy with AllOptical Ultrasound. In 2020 IEEE International Ultrasonics Symposium (IUS), pages 1-4, September 2020.

[3] Sacha Noimark, Richard J. Colchester, Ben J. Blackburn, et al. CarbonNanotube-PDMS Composite Coatings on Optical Fibers for All-Optical Ultrasound Imaging. Advanced Functional Materials, 26(46):83908396, 2016.

[4] James A. Guggenheim, Jing Li, Thomas J. Allen, et al. Ultrasensitive plano-concave optical microresonators for ultrasound sensing. Nature Photonics, 11(11):714-719, November 2017.

[5] George Dwyer, Richard J Colchester, Erwin J Alles, et al. Robotic Control of a Multi-Modal Rigid Endoscope Combining Optical Imaging with All-Optical Ultrasound. In 2019 International Conference on Robotics and Automation (ICRA), pages 3882-3888, May 2019.

[6] Richard J. Colchester, Callum Little, George Dwyer, et al. All-Optical Rotational Ultrasound Imaging. Scientific Reports, 9(1):5576, April 2019.

[7] Malcolm C Finlay, Charles A Mosse, Richard J Colchester, et al. Through-needle all-optical ultrasound imaging in vivo: a preclinical swine study. Light: Science \& Applications, 6(12):e17103, 2017.

[8] Fabrication of Refractive Index Tunable Polydimethylsiloxane Photonic Crystal for Biosensor Application. Physics Procedia, 19:146-151, January 2011.

[9] 0.22 NA Solarization-Resistant Step Index Multimode Optical Fibers. https://www.thorlabs.com/newgrouppage $9 . c f m$ ?objectgroup_id=6840 $\& \mathrm{pn}=\mathrm{UM} 22-300$. (accessed: Mar. 15, 2021).
[10] FOCUS - Michigan State University. https://www.egr.msu.edu/\%7Efultras-web/index.php. (accessed: Aug. 18, 2021).

[11] J. C. Lockwood and J. G. Willette. High-speed method for computing the exact solution for the pressure variations in the nearfield of a baffled piston. The Journal of the Acoustical Society of America, 53(3):735-741, March 1973.

[12] Peter R. Stepanishen. Transient Radiation from Pistons in an Infinite Planar Baffle. The Journal of the Acoustical Society of America, 49(5B):1629-1638, May 1971.

[13] Erwin J. Alles, Sacha Noimark, Efthymios Maneas, et al. Video-rate all-optical ultrasound imaging. Biomedical Optics Express, 9(8):34813494, August 2018.

[14] Richard S. C. Cobbold. Foundations of Biomedical Ultrasound. Oxford University Press, September 2006.

[15] Erwin J Alles, Richard J Colchester, and Adrien E Desjardins. Adaptive All-Optical Ultrasound Imaging Through Temporal Modulation of Excitation Light. In 2018 IEEE International Ultrasonics Symposium (IUS), pages 1-9, October 2018. 